\title{
Disappearing brain lesions, psychosis and epilepsy: a report of two cases
}

\author{
Anthony Feinstein, Maria Ron, Simon Wessely
}

\begin{abstract}
The association between disappearing brain lesions, psychosis and epilepsy in two patients is reported for the first time. Mechanisms to explain the underlying pathogenesis are postulated.
\end{abstract}

In primary generalised epilepsy, imaging procedures seldom detect structural abnormalities that correlate with areas of altered cerebral function. Thus Gastaut and Gastaut, ${ }^{1}$ reporting on 401 computed tomography (CT) scans in a sample of 500 consecutive patients with epilepsy, found $90^{\circ}{ }_{0}$ of their 73 patients with primary generalised epilepsy (idiopathic) to have normal brain scans. The percentage of positive scans, however, rose to $61^{\circ}{ }_{\circ}$ and $63^{\circ}{ }_{0}$ for secondary generalised and partial seizures respectively, the CT abnormalities reflecting the presence of demonstrable brain pathology, such as atrophy or space occupying lesions. More unusual is the presence of well demarcated, often contrast enhancing, lesions that appear during various types of ictal activity and disappear either spontaneously, or in response to the control of seizure activity brought about by anticonvulsant drugs alone, ${ }^{2-5}$ or with the addition of steroids. ${ }^{6}$

In the reports no mention has been made of any psychiatric symptomatology occurring in these patients. We report two such cases of disappearing lesions on $\mathrm{CT}$, who in addition to their seizures, presented with grossly abnormal mental states. To our knowledge, this is the first report linking psychosis, epilepsy and the disappearing lesions on CT.

Institute of Neurology, Queen Square, London A Feinstein

The National Hospital for Nervous Diseases, and Institute of Neurology, Queen Square, London MRon

The Institute of Psychiatry, De Crespigny Park, London

$\mathrm{S}$ Wessely

Correspondence to: Dr Anthony Feinstein, Institute of Neurology, Queen Square House, Queen Square, Londo WCIN 3BC, United Kingdom.

Received 27 June 1989 Accepted 6 September 1989
Case 1

A previously healthy 50 year old bus conductress with no past psychiatric history, gradually developed paranoid ideas with associated depressive features (anorexia, weight loss and disturbed sleep with early morning waking). She described voices commenting about her critically in the third person, and felt guilty about the allegations the voices made, namely that she was "dirty" and had had an extra marital affair. Her husband reported that during the previous two years she had become increasingly tense and withdrawn.

The patient's mother died giving birth but the patient herself did not suffer any obstetric complications. There was no documented history of mental illness in the family. Her upbringing and work record were uneventful.

She responded well to a period of inpatient psychiatric care during which perphenazine and a tricyclic antidepressant were prescribed. Full insight into her condition returned. A week after her discharge from hospital she again became deluded, while being treated for an upper respiratory tract infection. Ten days after this, she had two generalised convulsions. Neurological examination revealed no localising signs, but CT showed a contrast enhancing left fronto-temporal mass initially thought to be a tumour (fig 1A). She was given dexamethasone and phenytoin $100 \mathrm{mgm}$ three times a day and had no further epileptic attacks. Four weeks after the initial scan a repeat scan was normal (fig 1B), the dexamethasone was discontinued and her mental state returned to normal. However, four months later paranoid delusions returned. The patient believed her premises were bugged and that she was being persecuted. She was readmitted to hospital.

On examination, the only positive findings were a fine rotatory nystagmus on lateral gaze, more pronounced when looking to the right, and a mild gait ataxia. She appeared suspicious and tense but cooperative. Her speech was rapid with occasional loosening of associations and her mood was normal. Paranoid delusions of a persecutory nature, and auditory hallucinations in the third person were elicited. A Present State Examination (PSE) ${ }^{7}$ carried out retrospectively, gave a Catego subclass of "nuclear schizophrenia".

The results of the investigations were as follows: FBC, ESR, urea and electrolytes, LFT's, VDRL, serum phenytoin, T4, and $\mathrm{TSH}$ were all normal. A baseline prolactin was $2465 \mathrm{mU} / \mathrm{ml}$ (normal range $=118-500 \mathrm{mU} /$ $\mathrm{ml})$. Repeat CT showed no recurrence of the original lesion. An EEG showed a left temporal slow wave abnormality but no epileptiform activity. Psychometric testing revealed no significant change compared to 10 months earlier when she first became ill. She was functioning at the lower level of the normal IQ range and there was no evidence to suggest that this represented any intellectual decline.

She responded well to pimozide $20 \mathrm{mg}$ daily and her mental state again returned to normal. She has remained seizure free over the past eight years, and although mild paranoid symptoms have occasionally returned, these have not warranted admission. She has not taken medication for the past four years.

Case 2

A previously well, 22 year old unmarried hotel receptionist suddenly developed Jacksonian type seizures affecting the right side of her body. As the attacks increased in frequency, 

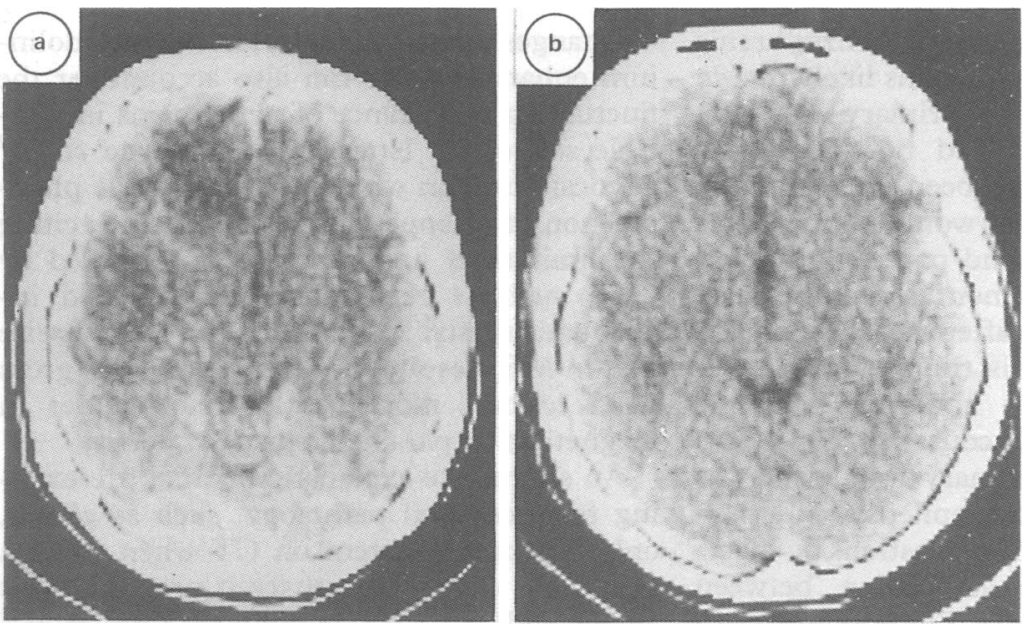

Fig $1 A$ Ring enhancing lesion in the left frontotemporal area seen on initial $C T$ (within 48 hours of seizure onset).

Fig $1 B$ Repeat $C T$ carried out one month after the first, showing resolution of the lesion.

Fig $2 A$ Ring enhancing lesion with surrounding. vasogenic oedema seen in the right tempero-parietal area, three weeks after the onset of symptoms.

Fig $2 B$ Repeat $C T$ scan carried out two months after the onset of symptoms, showing resolution of the lesion.
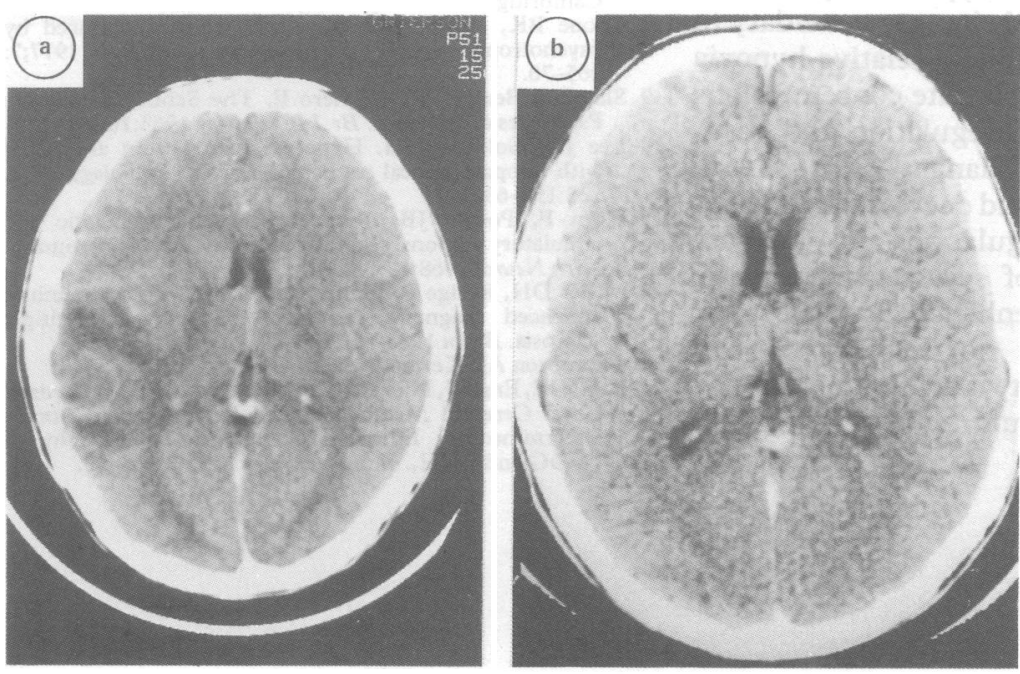

was labile. She referred to a variety of delusional beliefs, such as people playing tricks on her, dropping clues, removing her food, and leaving gold in her room. She also developed erotomania concerning a member of staff. She complained of somatic hallucinatory experiences involving electric shocks to the brain, legs, hips and pelvis. At this point she was given chlorpromazine. A PSE Catego subclass of "nuclear schizophrenia" (NS) was obtained.

The patient's general physical examination was consistently normal throughout. The following investigations were carried out and were all normal: FBC, ESR, urea and electrolytes, LFTs, serum B12 and folate, clotting profile, VDRL, thyroid function tests, CSF, autoantibodies, tests for Lyme disease, and viral titres for Toxoplasmosis, Toxocara, Listeria, Legionella and Mycoplasma. She was HIV negative.

She was treated with a combination of chlorpromazine and carbamazepine. Her seizures were clinically controlled although her EEG continued to show persistent background right sided abnormalities, with paroxysmal activity in the right posterior temporal region. Two follow up scans remained normal (fig $2 B$ ).

Over a period of three months her mental state became normal, although when placed under stress, auditory hallucinations transiently reappeared. She was eventually discharged on carbamazepine $200 \mathrm{mgms}$ four times a day. At follow up examination a year later, she had remained free from seizures and psychotic symptoms, although her boyfriend described her as prone to paroxysmal and violent outbursts.

\section{Discussion}

These two cases had a number of points in common. Both experienced epileptic seizures, had transient focal CT abnormalities and developed schizophrenic symptomatology in the absence of previous psychiatric illness. There were also a number of important differences between the two cases. The first patient, who was considerably older, presented with a paranoid state and prominent affective symptoms whereas in the second patient psychotic symptoms developed following epileptic activity. Seizure activity was also different, with generalised tonic-clonic seizures appearing in the first and partial complex seizures progressing to secondary generalised in the second.

The cause of epilepsy in our patients remains unclear. In the first case, the large increase in her neuroleptic medication in the week before the onset of her seizures may have played an important role. Toone and Fenton ${ }^{8}$ reported similar occurrences in 39 of their 41 psychiatric patients who developed generalised epileptic seizures de novo without localising signs. No such precipitating factor was present in the second patient. The severe disturbance in the level of consciouness, persistent seizures and EEG abnormalities point towards a primary organic insult, despite the difficulties in making a precise diagnosis. 
Although it is uncommon for schizophrenia to begin at the age of 50, it seems likely that in our first patient this was the primary diagnosis. The fact that she has had no subsequent seizures, and that she has been intermittently psychotic over the years, would support this assumption. In our second patient, however, the psychosis began two months after the onset of epilepsy and a month after the lesion on CT had disappeared. By this time, although her EEG remained grossly abnormal epileptic attacks had ceased. The course of her illness would suggest that the primary brain insult was responsible both for the epilepsy and psychosis, in a way similar to suggestions by Slater et $a l^{9}$ explaining the association between epilepsy and schizophrenia. The possibility of a steroid induced psychosis in our two patients was considered most unlikely, as the necessary temporal relationship between the psychosis and treatment was not present.

In neither of the two cases could an obvious cause be found to explain these disappearing lesions. In the first case, the aetiology was initially thought to be either vascular (for example, microvascular malformations, rupture of a small angioma), or the result of focal encephalitis, but the absence of any relevant clinical or laboratory findings and the rapid resolution of the lesion makes this unlikely. An encephalitic process was also considererd as a possibility in the second case, but thought unlikely to explain the well circumscribed CT lesion.

There remains the possibility that these transient CT findings could be the result, rather than the cause of the focal seizure activity. Similar conclusions have been reached by a number of previous studies which have reported disappearing $\mathrm{CT}^{2-46}$ and $\mathrm{MRI}^{5}$ lesions in patients with complex partial and generalised seizures in different age groups. In the largest series of cases reported, ${ }^{4}$ complete resolution of the lesion was observed in 37 of 46 patients, corresponding to seizure control, and taking place in most patients within 16 weeks.

A number of theories have been postulated to explain the transient nature of these disappearing lesions. Lesion enhancement probably represents a break in the blood-brain barrier (BBB) with the observed hypodensity accounted for by oedema. Local changes secondary to ictal activity would include a relative hypoxia with increased $\mathrm{CO} 2$ and lactate concentrations leading to a loss of autoregulation and vasodilatation. ${ }^{10}$ Systemic changes such as increased blood pressure and decreased blood $\mathrm{pH}$ further inhibit autoregulation and increase CBF. ${ }^{11}$ With control of seizure activity, the $\mathrm{BBB}$ is restored and enhancement subsides. CT abnormalities are not associated with severity of seizure activity ${ }^{2}$ and it is unclear why this phenomenon should occur in only a minority of epileptics.
Changes in the BBB as detected on Gadolinium enhanced MRI can also account for the fluctuating appearance of new lesions in multiple sclerosis. ${ }^{12}$ However, Thompson et al ${ }^{13}$ advocate caution when interpreting the phenomenon of disappearing lesions in MS, citing the limitations imposed by MRI (shared by CT), such as repositioning errors and inadequate spatial resolution. They emphasise that in MS, despite the disappearance of gross MRI lesions, more subtle abnormalities in magnetisation parameters usually persist.

An alternative explanation is that pre-existing focal cerebral pathology, such as gliosis, only becomes apparent on CT when oedema associated with ictal activity is present. ${ }^{4}$ This agrees with the PET findings of Kuhl et al, ${ }^{14}$ who have shown decreased local cerebral glucose utilisation in the interictal period in areas that appeared normal on CT. The gliosis theory, however, appears unlikely in our two patients as there was no history of previous brain insults.

In summary, two cases of epilepsy, psychosis and disappearing lesions on CT are presented. While at present the exact nature of the pathogenesis is unclear, future improvements in imaging techniques may explain more fully the mechanisms underlying this rare triad.

The authors thanks to Dr Trimble for drawing our attention to one of the patients and for allowing us to report the case.

1 Gastaut H, Gastaut JL. Computerised transverse axial tomography in epilepsy. Epilepsia 1976;17:325-36.

2 Jayakumar PN, Taly AB, Mohan PK. Transient computerised tomographic (CT) abnormalities following partial seizures. Acta Neurol Scand 1985;72:26-9.

3 Zegers de Beyl D, Hermanus N, Colle H, Goldman S. Focal seizures with reversible hypodensity on the CT scan. J Neurol Neurosurg Psychiatry 1985;48:187-8.

4 Goulatia RK, Verma A, Mishra NK, Ahuja GK. Disappearing CT lesions in Epilepsy. Epilepsia 1987;28:523-7.

5 Kramer RE, Luders H, Lesser RP, Weinstein MR, Dinner DS, Morris HH, Wyllie E. Transient Focal Abnormalities of Neuroimaging Studies During Focal Status Epilepticus. Epilepsia 1987;28:528-32.

6 Sethi PK, Kumar BR, Madan VS, Mohan V. Appearing and disappearing CT scan abnormalities and seizures. $J$ Neurol Neurosurg Psychiatry 1985;48:866-9.

7 Wing JK, Cooper JE, Sartorious N. The measurement and classification of psychiatric symptoms. Cambridge: Cambridge University Press, 1974:93-126.

8 Toone BK, Fenton GW. Epileptic seizures induced by psychotropic drugs. Psychological Medicine 1977;7: 265-70.

9 Slater E, Beard AW, Glithero E. The Schizophrenia-like Psychoses of Epilepsy. Br J Psychiatry 1963;109:95-150.

10 Lee JC, Goldberg HI. Hypervascular pattern associated with idiopathic focal status epilepticus. Radiology 1977; 125:159-63.

11 Plum F, Posner JB, Troy B. Cerebral metabolic and circulatory responses to induced convulsions in animals. Arch Neurol 1968;18:1-13.

12 Miller DH, Rudge P, Johnson G, et al. Serial gadolinium enhanced magnetic resonance imaging in multiple sclerosis. Brain 1988;111:927-39.

13 Thompson AJ, Kermode AG. Personal communication.

Kuhl DE, Engel J, Phelps ME, Selin C. Epileptic Patterns of Local Cerebral Metabolism and Perfusion in Humans Determined by Emission Computed Tomography of ${ }^{18}$ FDG and ${ }^{13} \mathrm{NH}_{3}$. Ann Neurology 1980;8:348-60. 